\title{
$\mathrm{AlCl}_{3}$ 催化活化 $\mathrm{sp}^{3}-\mathrm{C}-\mathrm{H}$ 键合成 3-氮杂芳基-3-羟基-2-羟吲哚
}

\author{
翟娇娇姚志刚徐凡* \\ (苏州大学材料与化学化工学部 苏州 215123)
}

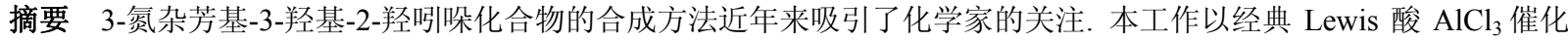
2-甲基吡啶化合物与靛红的加成反应，高效合成了 3 位氮杂芳基取代的 3-美基-2-羊弪吲哚化合物. 与已有报道相比，该方 法所用催化剂简单、易得、价廉，且催化剂用量较小，反应收率高. 同时，我们根据原位核磁的结果对该反应的机理进 行了推测.
\end{abstract}

关键词 3-着基-2-羟吲哚; 三氯化铝; 催化; C- $-\mathrm{H}$ 键活化

\section{Efficient Synthesis of 3-Azaarene-substituted 3-Hydroxy-2-oxindoles via Aluminium Chloride Catalyzed $\mathrm{sp}^{3}-\mathrm{C}-\mathrm{H}$ Functionalization}

\author{
Zhai, Jiaojiao Yao, Zhigang $\quad \mathrm{Xu}$, Fan* \\ (College of Chemistry, Chemical Engineering and Materials Science, Soochow University, Suzhou 215123)
}

\begin{abstract}
The synthesis of 3-azaarene-substituted 3-hydroxy-2-oxindoles has been receiving considerable attention in recent years. This paper describes an efficient method for the synthesis of 3-azaarene-substituted 3-hydroxy-2-oxindoles by aluminium chloride catalyzed reaction of 2-methylpyridines with isatins, which provides a practical and atom-economical process to afford this type of biologically important compounds in good yields using commercially available and inexpensive catalyst. A mechanism involving an aluminium chloride-catalyzed $\mathrm{sp}^{3}-\mathrm{C}-\mathrm{H}$ activation is proposed and the evidence of that is provided by in situ ${ }^{1} \mathrm{H}$ NMR.
\end{abstract}

Keywords 3-hydroxy-2-oxindole; aluminium chloride; catalysis; $\mathrm{C}-\mathrm{H}$ bond activation

3-羊基-2-羊至吲哚作为一种重要的结构单元广泛存 在于多种天然的或合成的生物活性物质中. 多项研究表 明, 含 3-着基-2-羟吲哚结构单元的化合物具有广谱生 物活性, 并已被成功地应用于抗癌、抗艾滋、抗氧化和 神经保护等领域 ${ }^{[1]}$. 令人感兴趣的是, 人们发现 3-羊基2-羊吲哚化合物的 C(3)位置的取代基在极大程度上决定 着化合物的生物特性, 因此, 化学家们开始着力发展带 有不同 $\mathrm{C}(3)$ 取代基的 3-羟基-2-羊弪吲哚化合物的合成方 法. 尽管有多个研究小组致力于这方面的研究, 也有相 当数量的 3-烷基或 3-芳基取代的此类化合物被合成出 来 ${ }^{[1,2]}$, 但关于 3 -氮杂芳基取代的 3-着基-2-羟吲哚化合 物的合成方法的报道却极为少见. 直到 2012 年, 几个研 究小组不约而同地各自报道了 3-氮杂芳基-3-羟基-2-羟 吲哚化合物的有效合成方法. Xiao 和 $\mathrm{Li}$ 等 ${ }^{[3]}$ 以 Brønsted 酸 HOTf 催化 2-甲基氮杂芳烃与靛红反应，合成一系列
3-羊基-2-羟吲哚化合物. 同时, Lewis 酸 $\mathrm{Yb}(\mathrm{OTf})_{3}$ 也被 发现能催化该反应的进行, 但活性并不突出 ${ }^{[4]}$. Lee 小组 ${ }^{[5]}$ 在催化量单质碘的存在下实现了同样的转化过程. 另

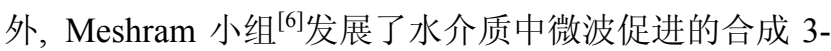
着基-2-羊吗哚化合物的类似方法. 上述文献报道均认 为反应是经由 2-甲基氮杂芳烃的苠基 $\mathrm{sp}^{3}-\mathrm{C}-\mathrm{H}$ 键活化 继而生成 $\mathrm{C}-\mathrm{C}$ 键而实现.

近年来, 通过 $\mathrm{C}-\mathrm{H}$ 键的活化从而实现其官能化的 合成策略因其高效和原子经济性的特点而备受瞩目 ${ }^{[7]}$, 而作为 $\mathrm{sp}^{3}-\mathrm{C}-\mathrm{H}$ 键活化领域的重要组成, 关于 2-甲基 氮杂芳烃苠位 $\mathrm{sp}^{3}-\mathrm{C}-\mathrm{H}$ 键活化的研究结果却并不丰 富 ${ }^{[8]}$. 在 3-氮杂芳基-3-羟基-2-差吲哚化合物的合成方 面, 虽然一些 Brønsted 酸 ${ }^{[3]}$ 和 Lewis 酸 ${ }^{[4,5]}$ 已被应用于催 化 2-甲基氮杂芳烃的苄基 $\mathrm{sp}^{3}-\mathrm{C}-\mathrm{H}$ 键活化以实现目标 分子的合成，但催化剂的用量均需达到 10 20 mol\%才

\footnotetext{
* E-mail: xufan@suda.edu.cn

Received February 28, 2014; revised April 7, 2014; published online April 16, 2014

Project supported by the National Natural Science Foundation of China (Nos. 21272168, 20872106).

国家自然科学基金(Nos. 21272168, 20872106)资助项目。
} 
能使反应顺利进行. 在我们课题组已有的 Lewis 酸催化 靛红的 3-位亲核加成反应的研究工作的基础上 ${ }^{[9]}$, 我们 考察了经济易得的 $\mathrm{AlCl}_{3}$ 作为 Lewis 酸催化剂在 2-甲基 吡啶化合物与靛红反应中的活性, 获得了较好的结果. 在我们的工作即将完成之时, Reddy 小组 ${ }^{[10]}$ 报道了在 PEG-400 中, 无催化剂条件下发生的甲基吡啶和靛红的 加成反应, 成功制备了 3-着基-3-(2-吡啶甲基)吲哚-2-酤 化合物. 这一方法条件温和、环境友好、令人瞩目. 尽 管如此, 我们仍希望在此报道我们的工作, 为 3-氮杂芳 烃取代的 3-差基-2-差吲哚化合物的合成提供另一种经 济、高效的途径.

\section{1 结果与讨论}

首先, 我们以 $3 \mathrm{~mol} \%$ 的 $\mathrm{AlCl}_{3}$ 催化 $N$-烯丙基靛红 (1a)与 2,6-二甲基吡啶 (2a)的反应为模板反应(表 1). 反 应在回流的甲苯溶剂中进行 $24 \mathrm{~h}$ 后(表 1, Entry 1), 以 70\%的收率得到了 1-烯丙基-3-羊基-3-[2-(6-甲基吡啶)甲 基]吲哚-2-酮(3a). 在通过常规的核磁共振、质谱、红外 等测试手段确认了产物结构之后, 我们对包括溶剂、温 度、反应时间及底物比在内的反应条件进行了优化. 从 表 1 可以看出, 在所笁选的溶剂中, 间二甲苯、氯苯和 $N, N$-二甲基甲酰胺(DMF)均给出了最好的结果(表 1 ,
Entries 2,6，8). 但是，由于间二甲苯中的反应总伴随着 较多的副反应，而氯苯众所周知并非是一个环境友好试 剂, 因此, 我们选择以 $N, N-$ 二甲基甲酰胺(DMF)作为反 应溶剂对反应作进一步的考察. 当反应温度由 $100{ }^{\circ} \mathrm{C}$ 升 高至 $110{ }^{\circ} \mathrm{C}$ 时，反应收率由 $30 \%$ 提高至 74\% (表 1, Entries 8,9), 但继续升温至 $140{ }^{\circ} \mathrm{C}$ 后收率反而下降. 另外, 对反应时间的考察表明, 反应进行 $8 \mathrm{~h}$ 后, 收率即能达 到最高(表 1, Entry 13). 所有的对照实验均表明，较低的 反应温度和较短的反应时间将导致反应的不完全，而过 高的温度和过长的时间则可能带来较多的副反应. 值得 注意的是，虽然产物是由靛红与 2,6-二甲基吡定以 $1 ： 1$ 的比例反应获得, 但若按此投料比操作, 产物收率相对 偏低(表 1, Entry 16)，仅为 53\%。但当反应体系中加入过 量的 2,6-二甲基吡啶后，产率有明显的提高(表 1, Entries 13，15). 该现象在其它 Brønsted 酸和 Lewis 酸催化的类 似反应中也有所报道 ${ }^{[3 \sim 5]}$. 这表明过量的 2,6-二甲基吡 啶对促进靛红转化为产物有重要作用, 但原因尚不可 知. 最后, 对反应浓度的考察结果显示当靛红的反应浓 度为 $2 \mathrm{~mol} / \mathrm{L}$ 时, 反应收率达到最佳(表 1, Entry 17).

我们进一步考察了不同的金属氯化物作为 Lewis 酸 对该反应的催化效果. 从表 2 中可以看出, 当不加入催 化剂时，反应收率仅为 $10 \%$ (表 2, Entry 1). $\mathrm{LaCl}_{3}, \mathrm{YbCl}_{3}$

表 $1 \mathrm{~N}$-烯丙基靛红与 2,6-二甲基吡啶反应条件的优化 ${ }^{a}$

Table 1 Condition screening for the reaction of $N$-allylisatin with 2,6-lutidine

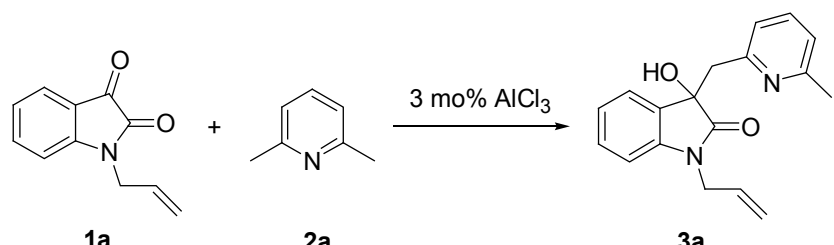

$1 \mathrm{a}$

$3 a$

\begin{tabular}{|c|c|c|c|c|c|c|}
\hline Entry & Solvent & Solvent loading $/ \mathrm{mL}$ & Temp. $/{ }^{\circ} \mathrm{C}$ & Time/h & $1 \mathbf{a}: 2 \mathbf{a}$ & Yield $/ \%$ \\
\hline 1 & Toluene & 1.0 & 110 & 24 & $1: 2.5$ & 70 \\
\hline 2 & $m$-Xylene & 1.0 & 110 & 24 & $1: 2.5$ & 74 \\
\hline 3 & Dioxane & 1.0 & 110 & 24 & $1: 2.5$ & 63 \\
\hline 4 & $\mathrm{EtOCH}_{2} \mathrm{CH}_{2} \mathrm{OEt}$ & 1.0 & 110 & 24 & $1: 2.5$ & 69 \\
\hline 5 & $n-\mathrm{BuOH}$ & 1.0 & 110 & 24 & $1: 2.5$ & 51 \\
\hline 6 & Chlorobenzene & 1.0 & 110 & 24 & $1: 2.5$ & 74 \\
\hline 7 & $\mathrm{Cl}_{2} \mathrm{CHCHCl}_{2}$ & 1.0 & 110 & 24 & $1: 2.5$ & Trace \\
\hline 8 & $\mathrm{DMF}$ & 1.0 & 110 & 24 & $1: 2.5$ & 74 \\
\hline 9 & DMF & 1.0 & 100 & 24 & $1: 2.5$ & 30 \\
\hline 10 & DMF & 1.0 & 120 & 24 & $1: 2.5$ & 75 \\
\hline 11 & DMF & 1.0 & 140 & 24 & $1: 2.5$ & 48 \\
\hline 12 & DMF & 1.0 & 110 & 12 & $1: 2.5$ & 76 \\
\hline 13 & DMF & 1.0 & 110 & 8 & $1: 2.5$ & 76 \\
\hline 14 & DMF & 1.0 & 110 & 6 & $1: 2.5$ & 67 \\
\hline 15 & DMF & 1.0 & 110 & 8 & $1: 1.2$ & 56 \\
\hline 16 & $\mathrm{DMF}$ & 1.0 & 110 & 8 & $1: 1.0$ & 53 \\
\hline 17 & DMF & 0.5 & 110 & 8 & $1: 2.5$ & 87 \\
\hline 18 & DMF & 0.2 & 110 & 8 & $1: 2.5$ & 70 \\
\hline
\end{tabular}

${ }^{a}$ Reactions conditions: $N$-allylisatin (1a) $(1 \mathrm{mmol})$ with $3 \mathrm{~mol} \% \mathrm{AlCl}_{3}$ relative to 1 a. 
以及 $\mathrm{ZnCl}_{2}$ 作为催化剂时反应的活性较差(表 2, Entries 2 4), 但当以 $\mathrm{FeCl}_{3}, \mathrm{CuCl}_{2}$ 或者 $\mathrm{SnCl}_{4}$ 催化该反应时却 能以较好的收率得到目标产物(表 2, Entries 5 7). $\mathrm{AlCl}_{3}$ 在所有被筛选的催化剂中活性最好, 因此我们对其用量 进行了进一步优化. 结果显示, 在 $\mathrm{AlCl}_{3}$ 的用量从 1 mol\%逐渐增加至 $5 \mathrm{~mol} \%$ (表 2, Entries 8 10)的过程中, 产物 3a 的收率呈现出先升高后降低的变化, 其中当 $\mathrm{AlCl}_{3}$ 的用量为 $3 \mathrm{~mol} \%$ 时反应达到最高收率 $87 \%$. 对粗 产物进行的分析显示过高的催化剂用量亦会导致副反 应的增多, 从而导致目标产物收率的下降. 最终我们确 定了该反应的最优条件是: 催化剂 $\mathrm{AlCl}_{3}$ 的用量为 3 $\mathrm{mol} \%$, 溶剂为 $\mathrm{DMF}$, 反应浓度为 $2 \mathrm{~mol} / \mathrm{L}$, 靛红与 2,6二甲基吡啶的投料比为 $1: 2.5$, 反应温度 $110{ }^{\circ} \mathrm{C}$, 反应 时间为 $8 \mathrm{~h}$.

表 $2 N$-烯丙基靛红与 2,6-二甲基吡啶反应催化剂的笁选 ${ }^{a}$

Table 2 Catalyst screening for the reaction of $N$-allylisatin with 2,6-lutidine

\begin{tabular}{ccc}
\hline Entry & Catalyst (mol\%) & Yield/\% \\
\hline 1 & - & 10 \\
2 & $\mathrm{LaCl}_{3}(3)$ & 30 \\
3 & $\mathrm{YbCl}_{3}(3)$ & 31 \\
4 & $\mathrm{ZnCl}_{2}(3)$ & 45 \\
5 & $\mathrm{FeCl}_{3}(3)$ & 76 \\
6 & $\mathrm{CuCl}_{2}(3)$ & 77 \\
7 & $\mathrm{SnCl}_{4}(3)$ & 80 \\
8 & $\mathrm{AlCl}_{3}(3)$ & 87 \\
9 & $\mathrm{AlCl}_{3}(1)$ & 60 \\
10 & $\mathrm{AlCl}_{3}(5)$ & 68 \\
\hline
\end{tabular}

${ }^{a}$ Reactions conditions: $N$-allylisatin (1a) $(1 \mathrm{mmol})$ and 2,6-lutidine (2a) $(2.5$ mmol) in DMF $(0.5 \mathrm{~mL})$ at $110{ }^{\circ} \mathrm{C}$ for $8 \mathrm{~h}$.

在上述最优条件下, 我们对一系列不同结构的靛红 的反应活性进行了考察, 结果列于表 3 中. 我们发现, 无论拝红的 $\mathrm{N}$ 上是被烷基、烯丙基还是苠基取代, 反应 均能以较高的收率制备得到相应的 3-羊基-2-羊弪弜哚化 合物. 另外, 无论靛红苯环上的取代基是在 4 位、 5 位还 是 7 位, 也无论是吸电子基团还是供电子基团, 反应均 能顺利地进行. 当以 2-甲基吡啶替代 2,6-二甲基吡啶作 为底物时, 反应效果也大致相似. 令人感兴趣的是, 当 以 2-甲基喹啉为底物时, 反应在标准条件下以 $53 \%$ 的收 率生成了两分子 2-甲基喹啉与靛红的加成产物(Eq. 1). 这一现象也曾出现在已有报道的 HOTf 以及 $\mathrm{Yb}(\mathrm{OTf})_{3}$ 催化的 2-甲基喹啉与责定红的反应中 ${ }^{[3,4]}$.

为了更深入地了解 $\mathrm{AlCl}_{3}$ 催化该反应的实质, 我们 利用原位核磁共振的方法对 $\mathrm{AlCl}_{3}$ 与 2-甲基吡啶相互作 用前后分子内特征质子的化学位移变化进行跟踪. 将 2甲基吡啶在氝苯中的 ${ }^{1} \mathrm{H}$ NMR 谱图(图 1, A)与 $\mathrm{AlCl}_{3}$ 和 2-甲基吡啶混合后在氝苯中的 ${ }^{1} \mathrm{H}$ NMR 谱图(图 1, B)对
表 $3 \mathrm{AlCl}_{3}$ 催化 2-甲基吡啶化合物与靛红的反应 ${ }^{a}$ Table $3 \quad \mathrm{AlCl}_{3}$-catalyzed reaction of 2-methylpyridines with various isatins

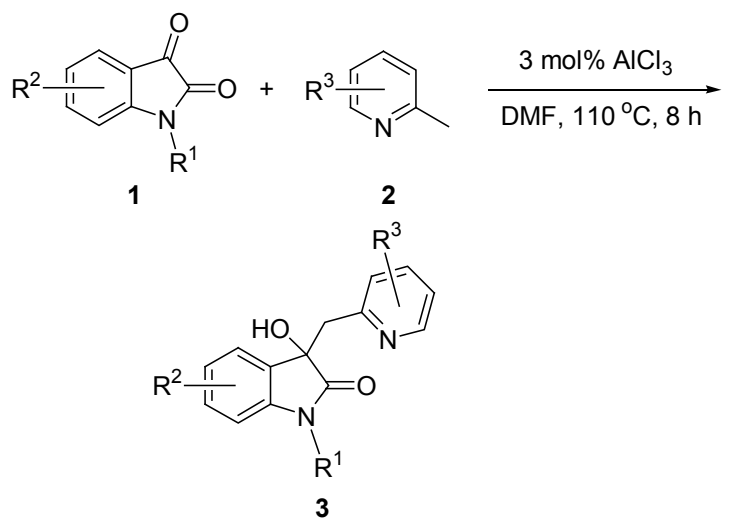

\begin{tabular}{cllccc}
\hline Entry & $\mathrm{R}^{1}$ & $\mathrm{R}^{2}$ & $\mathrm{R}^{3}$ & Product & Yield/\% \\
\hline 1 & Allyl & $\mathrm{H}$ & $6-\mathrm{Me}$ & $\mathbf{3 a}$ & 87 \\
2 & Et & $\mathrm{H}$ & $6-\mathrm{Me}$ & $\mathbf{3 b}$ & 77 \\
3 & $n$-Pr & $\mathrm{H}$ & $6-\mathrm{Me}$ & $\mathbf{3 c}$ & 79 \\
4 & Benzyl & $\mathrm{H}$ & $6-\mathrm{Me}$ & $\mathbf{3 d}$ & 74 \\
5 & Allyl & $4-\mathrm{Cl}$ & $6-\mathrm{Me}$ & $\mathbf{3 e}$ & 83 \\
6 & Allyl & $4-\mathrm{Br}$ & $6-\mathrm{Me}$ & $\mathbf{3 f}$ & 80 \\
7 & Allyl & $5-\mathrm{F}$ & $6-\mathrm{Me}$ & $\mathbf{3 g}$ & 73 \\
8 & Allyl & $5-\mathrm{Cl}$ & $6-\mathrm{Me}$ & $\mathbf{3 h}$ & 81 \\
9 & Allyl & $5-\mathrm{Br}$ & $6-\mathrm{Me}$ & $\mathbf{3 i}$ & 79 \\
10 & Allyl & $5-\mathrm{Me}$ & $6-\mathrm{Me}$ & $\mathbf{3 j}$ & 78 \\
11 & Allyl & $5-\mathrm{MeO}$ & $6-\mathrm{Me}$ & $\mathbf{3 k}$ & 76 \\
12 & Allyl & $7-\mathrm{F}$ & $6-\mathrm{Me}$ & $\mathbf{3 l}$ & 85 \\
13 & Allyl & $7-\mathrm{Cl}$ & $6-\mathrm{Me}$ & $\mathbf{3 m}$ & 79 \\
14 & Allyl & $\mathrm{H}$ & $\mathrm{H}$ & $\mathbf{3 n}$ & 79 \\
15 & Et & $\mathrm{H}$ & $\mathrm{H}$ & $\mathbf{3 o}$ & 76 \\
\hline
\end{tabular}

${ }^{a}$ Reactions conditions: isatin $(1 \mathrm{mmol})$ and 2-methylpyridine $(2.5 \mathrm{mmol})$ with $3 \mathrm{~mol} \% \mathrm{AlCl}_{3}$ relative to isatin in $0.5 \mathrm{~mL}$ of DMF at $110{ }^{\circ} \mathrm{C}$ for $8 \mathrm{~h}$.

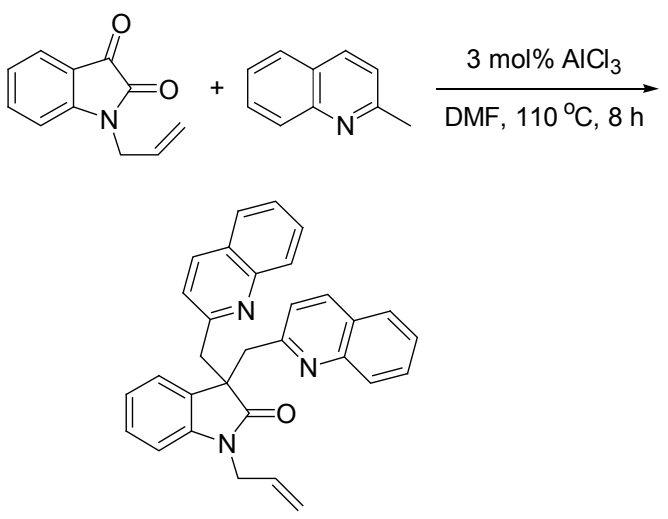

照可以发现，2-甲基吡啶中的甲基质子共振信号向低场 移动了约 $\delta 0.11$, 与此同时吡啶 6-位质子的共振信号也 向低场移动了约 $\delta 0.20$, 一致表明 $\mathrm{AlCl}_{3}$ 与 2-甲基吡啶中 的 $\mathrm{N}$ 原子发生了有效配位，从而活化了上述两处质子. 作为对照, $\mathrm{LaCl}_{3}$ 与 2-甲基吡啶混合后的 ${ }^{1} \mathrm{H} \mathrm{NMR}$ 谱图 (图 1, C)中并不能观察到 2-甲基吡啶化学位移的明显变 化, 这一现象与 $\mathrm{LaCl}_{3}$ 催化 2-甲基吡啶化合物与靛红反 
应的实际结果也是吻合的.

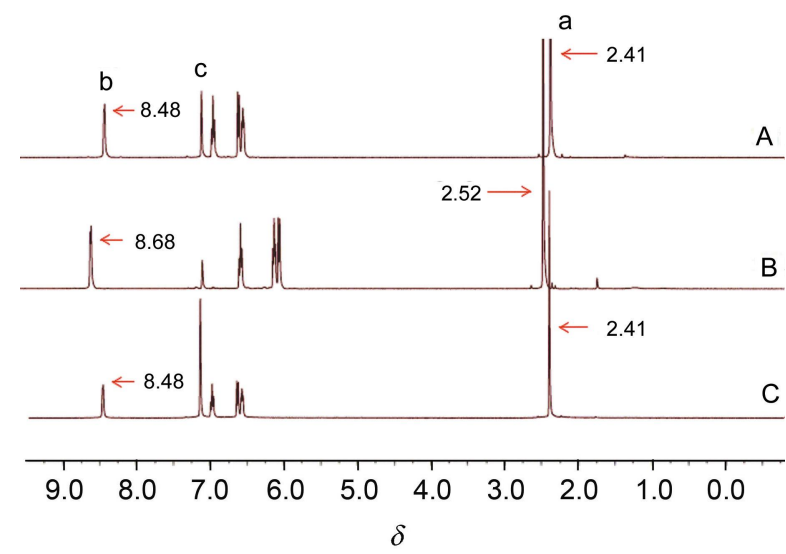

图 1 (A) 2-甲基吡啶、(B) 2-甲基吡啶 $+\mathrm{AlCl}_{3}$ 和(C) 2-甲基吡 啶 $+\mathrm{LaCl}_{3}$ 的 ${ }^{1} \mathrm{H} \mathrm{NMR}$ 谱图(気苯)

Figure $1 \quad{ }^{1} \mathrm{H}$ NMR spectra of (A) 2-methylpyridine, (B) [2-methylpyridine $\left.+\mathrm{AlCl}_{3}\right]$, and $(\mathrm{C})$ [2-methylpyridine $+\mathrm{LaCl}_{3}$ ] in benzene- $d_{6}$

(a) $\mathrm{CH}_{3}$ protons; (b) $\mathrm{H}-6$ proton of the pyridine; (c) benzene proton in benzene- $d_{6}$

综合上述结果并参考相关文献[4], 我们认为该反 应的机理(Scheme 1)包括三步过程: (i) $\mathrm{Al}^{3+}$ 与吡啶中的 氮配位生成活性中心 $\mathbf{A}$, 活化甲基 $\mathrm{C}-\mathrm{H}$ 键; (ii)苄基碳 对靛红 3 位羰基发生亲核进攻; (iii)所得中间体的 $\mathrm{Al}-\mathrm{O}$ 键发生质解，生成目标产物 $\mathbf{3}$, 同时再生活性中心 A. 机 理中活性中心 $\mathbf{A}$ 与靛红羰基的配位不仅活化了羰基，同 时也拉近了 2-甲基吡定和靛红的距离, 因此活性中心 $\mathbf{A}$ 的生成对于该反应的顺利进行至关重要.

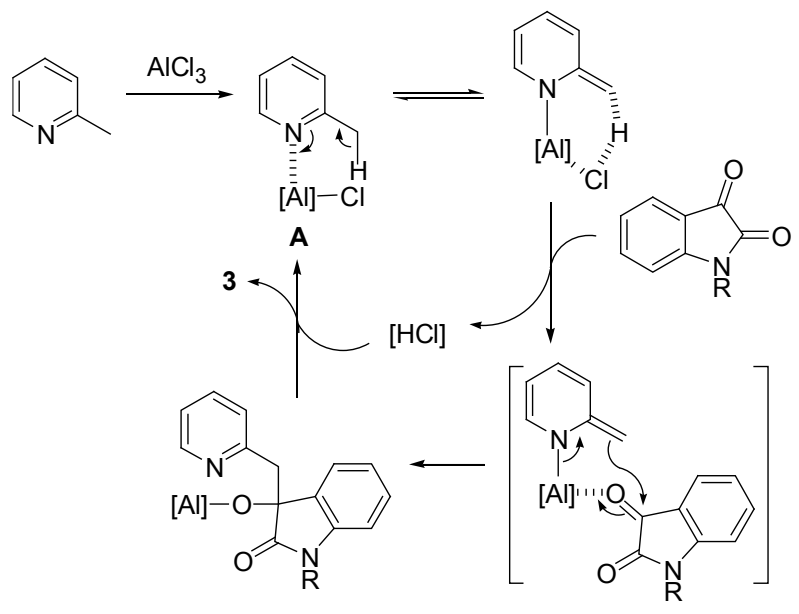

Scheme 1

\section{2 结论}

使用经典 Lewis 酸 $\mathrm{AlCl}_{3}$ 催化 2-甲基吡啶化合物与 静红的加成反应，高效地合成了 3-氮杂芳基取代的 3-羟
基-2-羊弪吲哚化合物. 反应所用催化剂简单易得，价格便 宜, 且所需催化剂用量小, 收率较高, 为 3-氮杂芳基-3差基-2-差哗哚化合物的合成提供了一个较为实用且具 有原子经济性的途径. 此外, 我们基于原位核磁的结果 对反应机理进行了推测, 认为反应的关键步骤是 $\mathrm{AlCl}_{3}$ 与吡啶配位引发的 $\mathrm{sp}^{3}-\mathrm{C}-\mathrm{H}$ 键活化.

\section{3 实验部分}

\section{1 仪器与试剂}

熔点由 SGW X-4 显微熔点仪测定, 温度计未校正; 核磁共振谱 $\left({ }^{1} \mathrm{H}\right.$ 或 $\left.{ }^{13} \mathrm{C}\right)$ 采用美国 Varian Unity-INOVA 400 核磁共振谱仪测定, 以 $\mathrm{CDCl}_{3}$ 为溶剂, TMS 为内标; 红外光谱采用 Scimitar 1000 型红外分光光度计测定; 质 谱采用美国 Agilent 1200/6220 型液相色谱一质谱联用仪 测定. 实验过程在氩气保护下采用 Schlenk 技术进行. 柱层析使用 300 400 目硅胶.

\section{2 实验方法}

3-氮杂芳基-3-差基-2-羟吲哚的一般合成步骤: 在 氩气保护下, 称取 $4 \mathrm{mg} \mathrm{AlCl} 3(0.03 \mathrm{mmol})$, 依次加入 2甲基吡啶化合物 $(2.5 \mathrm{mmol}) 、$ 靛红 $(1 \mathrm{mmol})$ 和 $0.5 \mathrm{~mL}$ $\mathrm{DMF}$ ，混合均匀后于 $110{ }^{\circ} \mathrm{C}$ 反应 $8 \mathrm{~h}$. 反应结束后加入 少量水, 用 $30 \mathrm{~mL}$ 乙酸乙酯萃取有机层 3 次. 有机相经 无水硫酸钠干燥后旋转蒸发除去低沸物, 所得粗产物经 硅胶柱层析 [淋洗剂: $V$ (石油醚)：V(乙酸乙酯 $)=7 ： 1$ ]得 到目标产物.

1-烯丙基-3-羟基-3-[2-(6-甲基吡啶)甲基]吲槑-2-酮 (3a): 白色固体, 产率 $87 \%$. m.p. 99 $100{ }^{\circ} \mathrm{C} ;{ }^{1} \mathrm{H}$ NMR $\left(\mathrm{CDCl}_{3}, 400 \mathrm{MHz}\right) \delta: 8.05(\mathrm{~s}, 1 \mathrm{H}), 7.54(\mathrm{t}, J=7.7 \mathrm{~Hz}, 1 \mathrm{H})$, $7.22(\mathrm{t}, J=7.8 \mathrm{~Hz}, 1 \mathrm{H}), 7.13(\mathrm{~d}, J=7.8 \mathrm{~Hz}, 1 \mathrm{H}), 6.92(\mathrm{t}$, $J=7.5 \mathrm{~Hz}, 1 \mathrm{H}), 6.85(\mathrm{~d}, J=7.6 \mathrm{~Hz}, 1 \mathrm{H}), 6.82 \sim 6.80(\mathrm{~m}$, $2 \mathrm{H}), 5.88 \sim 5.79(\mathrm{~m}, 1 \mathrm{H}), 5.36 \sim 5.20(\mathrm{~m}, 2 \mathrm{H}), 4.37(\mathrm{dd}$, $J=16.0,5.2 \mathrm{~Hz}, 1 \mathrm{H}), 4.25$ (dd, $J=16.0,5.2 \mathrm{~Hz}, 1 \mathrm{H}), 3.30$ (d, $J=14.8 \mathrm{~Hz}, 1 \mathrm{H}), 3.02$ (d, $J=14.8 \mathrm{~Hz}, 1 \mathrm{H}), 2.60$ (s, $3 \mathrm{H}) ;{ }^{13} \mathrm{C} \mathrm{NMR}\left(\mathrm{CDCl}_{3}, 100 \mathrm{MHz}\right) \delta: 176.6,157.3,156.9$, $142.1,137.5,131.4,131.3,129.2,124.0,122.7,122.0$, 121.6, 117.6, 109.2, 76.2, 42.5, 42.3, 24.4; IR (KBr) v: $3322,1707,1614,1590,1491,1465,1438,1383,1176$, 995, 772, $761 \mathrm{~cm}^{-1}$; HRMS (ESI) calcd for $\mathrm{C}_{18} \mathrm{H}_{19} \mathrm{~N}_{2} \mathrm{O}_{2}$ $[\mathrm{M}+\mathrm{H}]^{+}$295.1441, found 295.1452.

1-乙基-3-羟基-3-[2-(6-甲基吡啶)甲基]吲哚-2-酮 $(3 b)^{[3]}$ : 黄色固体, 产率 77\%. m.p. 109 110 ${ }^{\circ} \mathrm{C} ;{ }^{1} \mathrm{H}$ NMR $\left(\mathrm{CDCl}_{3}, 400 \mathrm{MHz}\right) \delta: 8.01(\mathrm{~s}, 1 \mathrm{H}), 7.54(\mathrm{t}, J=7.6 \mathrm{~Hz}, 1 \mathrm{H})$, 7.25 (t, $J=7.6 \mathrm{~Hz}, 1 \mathrm{H}), 7.13(\mathrm{~d}, J=7.6 \mathrm{~Hz}, 1 \mathrm{H}), 6.91(\mathrm{t}$, $J=7.6 \mathrm{~Hz}, 1 \mathrm{H}), 6.85(\mathrm{~d}, J=7.2 \mathrm{~Hz}, 1 \mathrm{H}), 6.83(\mathrm{~d}, J=7.2$ $\mathrm{Hz}, 1 \mathrm{H}), 6.76(\mathrm{~d}, J=7.2 \mathrm{~Hz}, 1 \mathrm{H}), 3.80 \sim 3.67(\mathrm{~m}, 2 \mathrm{H})$, 
3.30 (d, $J=14.8 \mathrm{~Hz}, 1 \mathrm{H}), 2.98$ (d, $J=14.8 \mathrm{~Hz}, 1 \mathrm{H}), 2.60$ (s, 3H), $1.26(\mathrm{t}, J=7.2 \mathrm{~Hz}, 3 \mathrm{H})$.

1-丙基-3-羊基-3-[2-(6-甲基吡啶)甲基]吲哚-2-酮 (3c $)^{[3]}$ : 黄色固体, 产率 79\%. m.p. 101 $102{ }^{\circ} \mathrm{C} ;{ }^{1} \mathrm{H}$ NMR $\left(\mathrm{CDCl}_{3}, 400 \mathrm{MHz}\right) \delta: 7.95(\mathrm{~s}, 1 \mathrm{H}), 7.54(\mathrm{t}, J=7.6 \mathrm{~Hz}, 1 \mathrm{H})$, $7.26 \sim 7.22(\mathrm{~m}, 1 \mathrm{H}), 7.12(\mathrm{~d}, J=7.6 \mathrm{~Hz}, 1 \mathrm{H}), 6.91(\mathrm{t}, J=$ $7.6 \mathrm{~Hz}, 1 \mathrm{H}), 6.85 \sim 6.79(\mathrm{~m}, 3 \mathrm{H}), 3.71 \sim 3.57(\mathrm{~m}, 2 \mathrm{H}), 3.28$ (d, $J=14.8 \mathrm{~Hz}, 1 \mathrm{H}), 2.99$ (d, $J=14.8 \mathrm{~Hz}, 1 \mathrm{H}), 2.60$ (s, $3 \mathrm{H}), 1.76 \sim 1.66(\mathrm{~m}, 2 \mathrm{H}), 0.96$ (t, $J=7.2 \mathrm{~Hz}, 3 \mathrm{H})$.

1-芐基-3-羟基-3-[2-(6-甲基吡啶)甲基]吲哚-2-酮 (3d) ${ }^{[3]}$ : 白色固体, 产率 $74 \%$. m.p. $175 \sim 176{ }^{\circ} \mathrm{C} ;{ }^{1} \mathrm{H}$ $\operatorname{NMR}\left(\mathrm{CDCl}_{3}, 400 \mathrm{MHz}\right) \delta: 8.14(\mathrm{~s}, 1 \mathrm{H}), 7.55$ (t, $J=7.8$ $\mathrm{Hz}, 1 \mathrm{H}), 7.33 \sim 7.26(\mathrm{~m}, 5 \mathrm{H}), 7.15 \sim 7.12(\mathrm{~m}, 2 \mathrm{H}), 6.91 \sim$ $6.86(\mathrm{~m}, 2 \mathrm{H}), 6.80(\mathrm{~d}, J=7.8 \mathrm{~Hz}, 1 \mathrm{H}), 6.69(\mathrm{~d}, J=7.8 \mathrm{~Hz}$, $1 \mathrm{H}), 4.95(\mathrm{~d}, J=16.0 \mathrm{~Hz}, 1 \mathrm{H}), 4.82(\mathrm{~d}, J=15.6 \mathrm{~Hz}, 1 \mathrm{H})$, 3.35 (d, $J=14.8 \mathrm{~Hz}, 1 \mathrm{H}), 3.06$ (d, $J=14.8 \mathrm{~Hz}, 1 \mathrm{H}), 2.61$ (s, 3H).

1-烯丙基-4-氯-3-羟基-3-[2-(6-甲基吡啶)甲基]吲哚2-酮(3e): 黄色固体, 产率 $83 \%$. m.p. $130 \sim 131{ }^{\circ} \mathrm{C} ;{ }^{1} \mathrm{H}$ NMR $\left(\mathrm{CDCl}_{3}, 400 \mathrm{MHz}\right) \delta: 7.78(\mathrm{~s}, 1 \mathrm{H}), 7.50$ (t, $J=7.8$ $\mathrm{Hz}, 1 \mathrm{H}), 7.18$ (t, $J=8.0 \mathrm{~Hz}, 1 \mathrm{H}), 7.06(\mathrm{~d}, J=7.8 \mathrm{~Hz}, 1 \mathrm{H})$, $6.99(\mathrm{~d}, J=8.0 \mathrm{~Hz}, 1 \mathrm{H}), 6.88(\mathrm{~d}, J=7.8 \mathrm{~Hz}, 1 \mathrm{H}), 6.68(\mathrm{~d}$, $J=7.8 \mathrm{~Hz}, 1 \mathrm{H}), 5.81 \sim 5.71(\mathrm{~m}, 1 \mathrm{H}), 5.20 \sim 5.15(\mathrm{~m}, 2 \mathrm{H})$, 4.27 (dd, $J=16.0,4.8 \mathrm{~Hz}, 1 \mathrm{H}), 4.20$ (dd, $J=16.0,4.8 \mathrm{~Hz}$, $1 \mathrm{H}), 3.91$ (d, $J=14.4 \mathrm{~Hz}, 1 \mathrm{H}), 3.00$ (d, $J=14.4 \mathrm{~Hz}, 1 \mathrm{H}$ ), $2.55(\mathrm{~s}, 3 \mathrm{H}) ;{ }^{13} \mathrm{C} \mathrm{NMR}\left(\mathrm{CDCl}_{3}, 100 \mathrm{MHz}\right) \delta: 176.3,157.0$, 156.1, 144.1, 137.1, 131.6, 130.9, 130.4, 126.5, 123.9, 121.6, 121.0, 117.6, 107.6, 77.1, 42.2, 40.2, 24.2; IR (KBr) $v: 3356,1703,1612,1590,1457,1432,1377,1177,1069$, 1007, 997, 775, $759 \mathrm{~cm}^{-1}$; HRMS (ESI) calcd for $\mathrm{C}_{18} \mathrm{H}_{18} \mathrm{ClN}_{2} \mathrm{O}_{2}[\mathrm{M}+\mathrm{H}]^{+}$329.1051, found 329.1054.

1-烯丙基-4-澳-3-羟基-3-[2-(6-甲基吡啶)甲基]吲哚2-酮(3f): 黄色固体, 产率 80\%. m.p. $140 \sim 141{ }^{\circ} \mathrm{C} ;{ }^{1} \mathrm{H}$ NMR $\left(\mathrm{CDCl}_{3}, 400 \mathrm{MHz}\right) \delta: 7.70$ (s, 1H), 7.50 (t, $J=7.8$ $\mathrm{Hz}, 1 \mathrm{H}), 7.19(\mathrm{~d}, J=8.0 \mathrm{~Hz}, 1 \mathrm{H}), 7.11(\mathrm{t}, J=8.0 \mathrm{~Hz}, 1 \mathrm{H})$, $7.05(\mathrm{~d}, J=7.8 \mathrm{~Hz}, 1 \mathrm{H}), 6.88(\mathrm{~d}, J=7.2 \mathrm{~Hz}, 1 \mathrm{H}), 6.72(\mathrm{~d}$, $J=7.6 \mathrm{~Hz}, 1 \mathrm{H}), 5.80 \sim 5.70(\mathrm{~m}, 1 \mathrm{H}), 5.20 \sim 5.15(\mathrm{~m}, 2 \mathrm{H})$, 4.26 (dd, $J=16.4,4.8 \mathrm{~Hz}, 1 \mathrm{H}), 4.19$ (dd, $J=16.4,4.8 \mathrm{~Hz}$, $1 \mathrm{H}), 4.01(\mathrm{~d}, J=14.4 \mathrm{~Hz}, 1 \mathrm{H}), 2.96(\mathrm{~d}, J=14.4 \mathrm{~Hz}, 1 \mathrm{H})$, 2.55 (s, $3 \mathrm{H}) ;{ }^{13} \mathrm{C} \mathrm{NMR}\left(\mathrm{CDCl}_{3}, 100 \mathrm{MHz}\right) \delta: 176.3,156.9$, $155.9,144.2,137.0,130.8,130.5,127.9,126.9,121.5$, 121.0, 119.5, 117.5, 108.0, 77.5, 42.0, 40.1, 24.1; IR (KBr) $v: 3347,1707,1607,1590,1454,1432,1419,1176,1069$, 1006, 997, 773, $759 \mathrm{~cm}^{-1}$; HRMS (ESI) calcd for $\mathrm{C}_{18} \mathrm{H}_{17} \mathrm{BrN}_{2} \mathrm{O}_{2} \mathrm{Na} \quad[\mathrm{M}+\mathrm{Na}]^{+}$395.0366, found 395.0365.
1-烯丙基-5-氟-3-羟基-3-[2-(6-甲基吡啶)甲基]吲哚2-䣳(3g): 黄色固体, 产率 73\%. m.p. $110 \sim 111{ }^{\circ} \mathrm{C} ;{ }^{1} \mathrm{H}$ NMR $\left(\mathrm{CDCl}_{3}, 400 \mathrm{MHz}\right) \delta: 8.17(\mathrm{~s}, 1 \mathrm{H}), 7.57$ (t, $J=7.6$ $\mathrm{Hz}, 1 \mathrm{H}), 7.15(\mathrm{~d}, J=8.0 \mathrm{~Hz}, 1 \mathrm{H}), 6.95 \sim 6.90(\mathrm{~m}, 1 \mathrm{H})$, $6.87(\mathrm{~d}, J=7.6 \mathrm{~Hz}, 1 \mathrm{H}), 6.75 \sim 6.72(\mathrm{~m}, 1 \mathrm{H}), 6.51(\mathrm{~d}, J=$ $8.0 \mathrm{~Hz}, 1 \mathrm{H}), 5.87 \sim 5.77(\mathrm{~m}, 1 \mathrm{H}), 5.25 \sim 5.22(\mathrm{~m}, 2 \mathrm{H})$, $4.39 \sim 4.33(\mathrm{~m}, 1 \mathrm{H}), 4.28 \sim 4.22(\mathrm{~m}, 1 \mathrm{H}), 3.34(\mathrm{~d}, J=14.8$ $\mathrm{Hz}, 1 \mathrm{H}), 2.97$ (d, $J=14.8 \mathrm{~Hz}, 1 \mathrm{H}), 2.61$ (s, 3H), ${ }^{13} \mathrm{C} \mathrm{NMR}$ $\left(\mathrm{CDCl}_{3}, 100 \mathrm{MHz}\right) \delta: 176.2,159.0,157.3,156.5,137.9$, $137.6,132.8,131.1,122.1,121.5,117.7,115.3,112.1$, $109.8,76.3,42.4,42.3,24.3$; IR (KBr) v: 3321, 1703, 1590, 1577, 1493, 1463, 1438, 1373, 1179, 1149, 988, 912, $889,818,771 \mathrm{~cm}^{-1}$; HRMS (ESI) calcd for $\mathrm{C}_{18} \mathrm{H}_{18} \mathrm{FN}_{2} \mathrm{O}_{2}$ $[\mathrm{M}+\mathrm{H}]^{+}$313.1347, found 313.1347.

1-烯丙基-5-氯-3-羟基-3-[2-(6-甲基吡啶)甲基]吲哚2-酮(3h): 黄色固体, 产率 $81 \%$. m.p. $109 \sim 110{ }^{\circ} \mathrm{C} ;{ }^{1} \mathrm{H}$ NMR $\left(\mathrm{CDCl}_{3}, 400 \mathrm{MHz}\right) \delta: 8.18$ (s, 1H), 7.57 (t, $J=7.6$ $\mathrm{Hz}, 1 \mathrm{H}), 7.21 \sim 7.15(\mathrm{~m}, 2 \mathrm{H}), 6.87(\mathrm{~d}, J=7.6 \mathrm{~Hz}, 1 \mathrm{H})$, $6.77 \sim 6.73(\mathrm{~m}, 2 \mathrm{H}), 5.86 \sim 5.76(\mathrm{~m}, 1 \mathrm{H}), 5.24 \sim 5.20(\mathrm{~m}$, $2 \mathrm{H}), 4.35$ (dd, $J=16.4,4.0 \mathrm{~Hz}, 1 \mathrm{H}), 4.24(\mathrm{dd}, J=16.0,3.6$ Hz, 1H), 3.29 (d, $J=14.8 \mathrm{~Hz}, 1 \mathrm{H}), 3.00$ (d, $J=14.8 \mathrm{~Hz}$, $1 \mathrm{H}), 2.61(\mathrm{~s}, 3 \mathrm{H}) ;{ }^{13} \mathrm{C} \mathrm{NMR}\left(\mathrm{CDCl}_{3}, 100 \mathrm{MHz}\right) \delta: 176.0$, $157.0,156.1,140.4,137.4,132.6,130.8,128.8,127.7$, 124.3, 121.9, 121.4, 117.6, 110.1, 76.0, 42.2, 42.1, 24.1; IR (KBr) $v: 3311,1706,1593,1577,1483,1459,1436,1372$, $1177,1105,999,804,775 \mathrm{~cm}^{-1}$; HRMS (ESI) calcd for $\mathrm{C}_{18} \mathrm{H}_{18} \mathrm{ClN}_{2} \mathrm{O}_{2}[\mathrm{M}+\mathrm{H}]^{+}$329.1051, found 329.1054.

1-烯丙基-5-溴-3-羊基-3-[2-(6-甲基吡啶)甲基]吲哚2-酩(3i): 黄色固体, 产率 79\%. m.p. $127 \sim 128{ }^{\circ} \mathrm{C} ;{ }^{1} \mathrm{H}$ NMR $\left(\mathrm{CDCl}_{3}, 400 \mathrm{MHz}\right) \delta: 8.11(\mathrm{~s}, 1 \mathrm{H}), 7.56(\mathrm{t}, J=7.2$ $\mathrm{Hz}, 1 \mathrm{H}), 7.33$ (d, $J=8.0 \mathrm{~Hz}, 1 \mathrm{H}), 7.14(\mathrm{~d}, J=7.6 \mathrm{~Hz}, 1 \mathrm{H})$, $6.91 \sim 6.87(\mathrm{~m}, 2 \mathrm{H}), 6.69(\mathrm{~d}, J=8.0 \mathrm{~Hz}, 1 \mathrm{H}), 5.82 \sim 5.75$ (m, $1 \mathrm{H}), 5.22 \sim 5.18(\mathrm{~m}, 2 \mathrm{H}), 4.34 \sim 4.20(\mathrm{~m}, 2 \mathrm{H}), 3.28(\mathrm{~d}$, $J=14.4 \mathrm{~Hz}, 1 \mathrm{H}), 3.02$ (d, $J=14.8 \mathrm{~Hz}, 1 \mathrm{H}), 2.59$ (s, 3H); ${ }^{13} \mathrm{C} \mathrm{NMR}\left(\mathrm{CDCl}_{3}, 100 \mathrm{MHz}\right) \delta: 175.9,157.1,156.1,141.0$, $137.4,133.0,131.8,130.8,127.2,122.0,121.4,117.7$, 115.1, 110.6, 75.9, 42.3, 42.1, 24.2; IR (KBr) v: 3315, 1706, 1612, 1592, 1459, 1435, 1176, 1001, 989, 809, 774 $\mathrm{cm}^{-1}$; HRMS (ESI) calcd for $\mathrm{C}_{18} \mathrm{H}_{17} \mathrm{BrN}_{2} \mathrm{O}_{2} \mathrm{Na}[\mathrm{M}+\mathrm{Na}]^{+}$ 395.0366, found 395.0374.

1-烯丙基-5-甲基-3-羟基-3-[2-(6-甲基吡啶)甲基]吲 哚-2-酮(3j): 黄色固体, 产率 78\%. m.p. $69 \sim 70{ }^{\circ} \mathrm{C} ;{ }^{1} \mathrm{H}$ NMR $\left(\mathrm{CDCl}_{3}, 400 \mathrm{MHz}\right) \delta: 8.00(\mathrm{~s}, 1 \mathrm{H}), 7.55$ (t, $J=7.6$ $\mathrm{Hz}, 1 \mathrm{H}), 7.13(\mathrm{~d}, J=7.6 \mathrm{~Hz}, 1 \mathrm{H}), 7.03(\mathrm{~d}, J=7.6 \mathrm{~Hz}, 1 \mathrm{H})$, $6.86(\mathrm{~d}, J=7.6 \mathrm{~Hz}, 1 \mathrm{H}), 6.71 \sim 6.68(\mathrm{~m}, 2 \mathrm{H}), 5.87 \sim 5.77$ 
$(\mathrm{m}, 1 \mathrm{H}), 5.24 \sim 5.19(\mathrm{~m}, 2 \mathrm{H}), 4.34(\mathrm{dd}, J=16.4,4.8 \mathrm{~Hz}$, $1 \mathrm{H}), 4.23$ (dd, $J=16.4,4.8 \mathrm{~Hz}, 1 \mathrm{H}), 3.24$ (d, $J=14.4 \mathrm{~Hz}$, $1 \mathrm{H}), 3.06$ (d, $J=14.4 \mathrm{~Hz}, 1 \mathrm{H}), 2.60(\mathrm{~s}, 3 \mathrm{H}), 2.21$ (s, 3H); ${ }^{13} \mathrm{C} \mathrm{NMR}\left(\mathrm{CDCl}_{3}, 100 \mathrm{MHz}\right) \delta: 176.4,156.9,156.6,139.4$, $137.2,131.9,131.2,130.9,129.2,124.6,121.7,121.4$, $117.2,108.7,76.0,42.5,42.0,24.1,20.9$; IR (KBr) $v$ : 3318, 1698, 1605, 1591, 1496, 1458, 1434, 1419, 1368, 1160, 995, 802, $774 \mathrm{~cm}^{-1}$; HRMS (ESI) calcd for $\mathrm{C}_{19} \mathrm{H}_{20} \mathrm{~N}_{2} \mathrm{O}_{2} \mathrm{Na}[\mathrm{M}+\mathrm{Na}]^{+}$331.1417, found 331.1419.

1-烯丙基-5-甲氧基-3-羟基-3-[2-(6-甲基吡啶)甲基] 吲哚-2-酮(3k): 红色固体, 产率 76\%. m.p. 100 100.5 ${ }^{\circ} \mathrm{C} ;{ }^{1} \mathrm{H} \mathrm{NMR}\left(\mathrm{CDCl}_{3}, 400 \mathrm{MHz}\right) \delta: 8.08(\mathrm{~s}, 1 \mathrm{H}), 7.56(\mathrm{t}$, $J=7.6 \mathrm{~Hz}, 1 \mathrm{H}), 7.13(\mathrm{~d}, J=7.6 \mathrm{~Hz}, 1 \mathrm{H}), 6.87$ (d, $J=7.6$ $\mathrm{Hz}, 1 \mathrm{H}), 6.76 \sim 6.71(\mathrm{~m}, 2 \mathrm{H}), 6.42(\mathrm{~s}, 1 \mathrm{H}), 5.87 \sim 5.78(\mathrm{~m}$, $1 \mathrm{H}), 5.25 \sim 5.20(\mathrm{~m}, 2 \mathrm{H}), 4.35(\mathrm{dd}, J=16.4,4.8 \mathrm{~Hz}, 1 \mathrm{H})$, 4.24 (dd, $J=16.4,4.8 \mathrm{~Hz}, 1 \mathrm{H}), 3.66$ (s, 3H), 3.31 (d, $J=$ $14.8 \mathrm{~Hz}, 1 \mathrm{H}), 3.01$ (d, $J=14.8 \mathrm{~Hz}, 1 \mathrm{H}), 2.61(\mathrm{~s}, 3 \mathrm{H}) ;{ }^{13} \mathrm{C}$ NMR $\left(\mathrm{CDCl}_{3}, 100 \mathrm{MHz}\right) \delta: 176.2,157.0,156.6,155.7$, $137.3,135.2,132.2,131.3,121.8,121.5,117.4,113.4$, $111.0,109.5,76.3,55.5,42.5,42.2,24.2$; IR (KBr) $v$ : $3323,1697,1642,1593,1578,1491,1458,1434,1407$, 1381, 1270, 1181, 1091, 1031, 884, 816, $756 \mathrm{~cm}^{-1}$; HRMS (ESI) calcd for $\mathrm{C}_{19} \mathrm{H}_{20} \mathrm{~N}_{2} \mathrm{O}_{3} \mathrm{Na}[\mathrm{M}+\mathrm{Na}]^{+} 347.1366$, found 347.1370 .

1-烯丙基-7-氟-3-差基-3-[2-(6-甲基吡啶)甲基]吲哚2-酩(3I): 白色固体, 产率 85\%. m.p. 93 94 ${ }^{\circ} \mathrm{C} ;{ }^{1} \mathrm{H}$ NMR $\left(\mathrm{CDCl}_{3}, 400 \mathrm{MHz}\right) \delta: 8.22(\mathrm{~s}, 1 \mathrm{H}), 7.56(\mathrm{t}, J=7.6 \mathrm{~Hz}, 1 \mathrm{H})$, $7.14(\mathrm{~d}, J=7.6 \mathrm{~Hz}, 1 \mathrm{H}), 7.01 \sim 6.96(\mathrm{~m}, 1 \mathrm{H}), 6.90 \sim 6.85$ $(\mathrm{m}, 2 \mathrm{H}), 6.61(\mathrm{~d}, J=7.2 \mathrm{~Hz}, 1 \mathrm{H}), 5.96 \sim 5.87(\mathrm{~m}, 1 \mathrm{H})$, $5.25 \sim 5.16(\mathrm{~m}, 2 \mathrm{H}), 4.45 \sim 4.43(\mathrm{~m}, 2 \mathrm{H}), 3.28(\mathrm{~d}, J=14.8$ $\mathrm{Hz}, 1 \mathrm{H}), 3.02$ (d, $J=14.8 \mathrm{~Hz}, 1 \mathrm{H}), 2.60$ (s, 3H); ${ }^{13} \mathrm{C} \mathrm{NMR}$ $\left(\mathrm{CDCl}_{3}, 100 \mathrm{MHz}\right) \delta: 176.2,157.1,156.4,147.2,137.4$, $134.1,132.0,128.4,123.3,121.7,119.8,119.7,117.2$, 117.0, 76.2, 43.7, 42.4, 24.2; IR (KBr) v: 3201, 1727, 1632 , 1596, 1575, 1488, 1462, 1426, 1371, 1242, 1186, $774,754 \mathrm{~cm}^{-1}$; HRMS (ESI) calcd for $\mathrm{C}_{18} \mathrm{H}_{18} \mathrm{FN}_{2} \mathrm{O}_{2}[\mathrm{M}+$ $\mathrm{H}]^{+}$313.1347, found 313.1351 .

1-烯丙基-7-氯-3-差基-3-[2-(6-甲基吡啶)甲基]吲哚2-酠(3m): 白色固体, 产率 79\%. m.p. 70 71 ${ }^{\circ} \mathrm{C} ;{ }^{1} \mathrm{H}$ NMR $\left(\mathrm{CDCl}_{3}, 400 \mathrm{MHz}\right) \delta: 8.22(\mathrm{~s}, 1 \mathrm{H}), 7.56$ (t, $J=7.6$ $\mathrm{Hz}, 1 \mathrm{H}), 7.19$ (d, $J=8.0 \mathrm{~Hz}, 1 \mathrm{H}), 7.14$ (d, $J=7.6 \mathrm{~Hz}, 1 \mathrm{H})$, $6.89 \sim 6.84(\mathrm{~m}, 2 \mathrm{H}), 6.77(\mathrm{~d}, J=7.2 \mathrm{~Hz}, 1 \mathrm{H}), 6.01 \sim 5.91$ $(\mathrm{m}, 1 \mathrm{H}), 5.18 \sim 5.13(\mathrm{~m}, 2 \mathrm{H}), 4.70 \sim 4.69(\mathrm{~m}, 2 \mathrm{H}), 3.23(\mathrm{~d}$, $J=14.8 \mathrm{~Hz}, 1 \mathrm{H}), 3.03$ (d, $J=14.8 \mathrm{~Hz}, 1 \mathrm{H}), 2.60$ (s, 3H); ${ }^{13} \mathrm{C} \mathrm{NMR}\left(\mathrm{CDCl}_{3}, 100 \mathrm{MHz}\right) \delta: 176.9,156.9,156.1,137.7$,
$137.3,133.9,132.6,131.4,123.4,122.4,121.9,121.4$, $116.1,115.0,75.3,43.0,42.5,24.1$; IR (KBr) v: 3335, 1709, 1590, 1578, 1461, 1426, 1418, 1378, 1178, 1071, 1030, 755, $738 \mathrm{~cm}^{-1}$; HRMS (ESI) calcd for $\mathrm{C}_{18} \mathrm{H}_{18} \mathrm{Cl}-$ $\mathrm{N}_{2} \mathrm{O}_{2}[\mathrm{M}+\mathrm{H}]^{+}$329.1051, found 329.1050.

1-烯丙基-3-差基-3-(2-吡啶甲基)吲哚-2-酮(3n) ${ }^{[6]}$ : 白色固体，产率 79\%. m.p. 129 130 ${ }^{\circ} \mathrm{C} ;{ }^{1} \mathrm{H} \mathrm{NMR}$ $\left(\mathrm{CDCl}_{3}, 400 \mathrm{MHz}\right) \delta: 8.58(\mathrm{~s}, 1 \mathrm{H}), 7.67 \sim 7.49(\mathrm{~m}, 2 \mathrm{H})$, $7.28 \sim 7.21(\mathrm{~m}, 2 \mathrm{H}), 7.06(\mathrm{~d}, J=7.6 \mathrm{~Hz}, 1 \mathrm{H}), 6.93(\mathrm{t}, J=$ $7.6 \mathrm{~Hz}, 1 \mathrm{H}), 6.84 \sim 6.79(\mathrm{~m}, 2 \mathrm{H}), 5.87 \sim 5.77(\mathrm{~m}, 1 \mathrm{H})$, $5.35 \sim 5.20(\mathrm{~m}, 2 \mathrm{H}), 4.37(\mathrm{dd}, J=16.4,5.2 \mathrm{~Hz}, 1 \mathrm{H}), 4.23$ (dd, $J=16.0,5.2 \mathrm{~Hz}, 1 \mathrm{H}), 3.35$ (d, $J=14.8 \mathrm{~Hz}, 1 \mathrm{H}), 3.12$ (d, $J=14.8 \mathrm{~Hz}, 1 \mathrm{H})$.

1-乙基-3-差基-3-(2-吡啶甲基)吲哚-2-酥(3o): 白色 固体, 产率 76\%. m.p. $124 \sim 124.5{ }^{\circ} \mathrm{C} ;{ }^{1} \mathrm{H} \mathrm{NMR}\left(\mathrm{CDCl}_{3}\right.$, $400 \mathrm{MHz}) \delta: 8.58(\mathrm{~s}, 1 \mathrm{H}), 7.67 \sim 7.47(\mathrm{~m}, 2 \mathrm{H}), 7.29 \sim 7.23$ $(\mathrm{m}, 2 \mathrm{H}), 7.05(\mathrm{~d}, J=7.6 \mathrm{~Hz}, 1 \mathrm{H}), 6.92(\mathrm{t}, J=7.6 \mathrm{~Hz}, 1 \mathrm{H})$, $6.83 \sim 6.80(\mathrm{~m}, 2 \mathrm{H}), 3.80 \sim 3.66(\mathrm{~m}, 2 \mathrm{H}), 3.34(\mathrm{~d}, J=14.8$ $\mathrm{Hz}, 1 \mathrm{H}), 3.08$ (d, $J=14.4 \mathrm{~Hz}, 1 \mathrm{H}), 1.25$ (t, $J=7.2 \mathrm{~Hz}, 3 \mathrm{H})$; ${ }^{13} \mathrm{C} \mathrm{NMR}\left(\mathrm{CDCl}_{3}, 100 \mathrm{MHz}\right) \delta: 176.3,157.2,148.0,141.7$, $136.8,130.8,129.1,124.5,123.9,122.3,122.1,108.2$, 76.0, 43.0, 34.4, 12.3; IR (KBr) v: 3268, 2976, 1698, 1592, 1572, 1493, 1469, 1376, 1199, $755 \mathrm{~cm}^{-1}$; HRMS (ESI) calcd for $\mathrm{C}_{16} \mathrm{H}_{16} \mathrm{~N}_{2} \mathrm{O}_{2} \mathrm{Na}[\mathrm{M}+\mathrm{Na}]^{+}$291.1104, found 291.1104 .

1-烯丙基-3,3-二(2-喹啉甲基)吲哚-2-酮：黄色固体， 产率 53\%. m.p. 101.5 102.5 ${ }^{\circ} \mathrm{C} ;{ }^{1} \mathrm{H}$ NMR $\left(\mathrm{CDCl}_{3}, 400\right.$ $\mathrm{MHz}) \delta: 7.86 \sim 7.83(\mathrm{~m}, 4 \mathrm{H}), 7.65(\mathrm{~d}, J=8.0 \mathrm{~Hz}, 2 \mathrm{H}), 7.57$ (t, $J=7.6 \mathrm{~Hz}, 2 \mathrm{H}), 7.40$ (t, $J=7.2 \mathrm{~Hz}, 2 \mathrm{H}), 7.16(\mathrm{~d}, J=7.2$ $\mathrm{Hz}, 1 \mathrm{H}), 7.08$ (d, $J=8.8 \mathrm{~Hz}, 2 \mathrm{H}), 6.93$ (t, $J=7.2 \mathrm{~Hz}, 1 \mathrm{H})$, $6.84(\mathrm{t}, J=7.2 \mathrm{~Hz}, 1 \mathrm{H}), 6.40(\mathrm{~d}, J=7.6 \mathrm{~Hz}, 1 \mathrm{H}), 5.48 \sim$ $5.38(\mathrm{~m}, 1 \mathrm{H}), 4.80 \sim 4.69(\mathrm{~m}, 2 \mathrm{H}), 4.16(\mathrm{~d}, J=5.2 \mathrm{~Hz}$, 2H), 3.79 (d, $J=13.6 \mathrm{~Hz}, 2 \mathrm{H}), 3.69$ (d, $J=14.0 \mathrm{~Hz}, 2 \mathrm{H})$; ${ }^{13} \mathrm{C} \mathrm{NMR}\left(\mathrm{CDCl}_{3}, 100 \mathrm{MHz}\right) \delta: 178.6,157.1,147.5,143.0$, $135.4,131.5,130.3,129.1,127.6,127.3,126.7,125.9$, 124.6, 122.4, 121.6, 117.0, 109.8, 108.3, 53.4, 46.0, 42.4; IR (KBr) v: 3446, 1702, 1646, 1612, 1599, 1505, 1467, 1427, 1313, 1183, 1174, $757 \mathrm{~cm}^{-1}$; HRMS (ESI) calcd for $\mathrm{C}_{31} \mathrm{H}_{25} \mathrm{~N}_{3} \mathrm{ONa}[\mathrm{M}+\mathrm{Na}]^{+} 478.1890$, found 478.1892 .

\section{References}

[1] (a) Tokunaga, T.; Hume, W. E.; Nagamine, J.; Kawamura, T.; Taiji, M.; Nagata, R. Bioorg. Med. Chem. Lett. 2005, 15, 1789.

(b) Yong, S. R.; Ung, A. T.; Pyne, S. G.; Skelton, B. W.; White, A. H. Tetrahedron 2007, 63, 5579.

(c) Kohno, J.; Koguchi, Y.; Nishio, M.; Nakao, K.; Kuroda, M.; Shimizu, R.; Ohnuki, T.; Komatsubara, S. J. Org. Chem. 2000, 65, 
990.

(d) Tang, Y. Q.; Sattler, I.; Thiericke, R.; Grabley, S.; Feng, X. Z. Eur. J. Org. Chem. 2001, 261.

(e) Gan, C. Y.; Kam, T. S. Tetrahedron Lett. 2009, 50, 1059.

(f) Tokunaga, T.; Hume, W. E.; Umezome, T.; Okazaki, K.; Ueki, Y.; Kumagai, K.; Hourai, S.; Nagamine, J.; Seki, H.; Taiji, M.; Noguchi, H.; Nagata, R. J. Med. Chem. 2001, 44, 4641.

(g) Suzuki, H.; Morita, H.; Shiro, M.; Kobayashi, J. Tetrahedron 2004, 60, 2489.

(h) Peddibhotla, S. Curr. Bioact. Compd. 2009, 5, 20.

[2] (a) Rasmussen, H. B.; MacLeod, J. K. J. Nat. Prod. 1997, 60, 1152.

(b) Garden, S. J.; Torres, J. C.; Ferreira, A. A.; Silva, R. B.; Pinto, A. C. Tetrahedron Lett. 1997, 38, 1501.

(c) Miah, S.; Moody, C. J.; Richards, I. C.; Slawin, A. M. Z. J. Chem. Soc., Perkin Trans. 1 1997, 2405.

(d) Jnaneshwara, G. K.; Bedekar, A. V.; Deshpande, V. H. Synth. Commun. 1999, 29, 3627.

(e) Jnaneshwar, G. K.; Deshpande, V. H. J. Chem. Res., Synop. 1999, 632 .

(f) Suchy, M.; Kutschy, P.; Monde, K.; Goto, H.; Harada, N.; Takasugi, M.; Dzurilla, M.; Balentova, E. J. Org. Chem. 2001, 66, 3940.

[3] Niu, R.; Xiao, J.; Liang, T.; Li, X. W. Org. Lett. 2012, 14, 676.

[4] Niu, R.; Yang, S. Y.; Xiao, J.; Liang, T.; Li, X. W. Chin. J. Catal. 2012, 33, 1636.

[5] Vuppalapati, S. V. N.; Lee, Y. R. Tetrahedron 2012, 68, 8286.

[6] Meshram, H. M.; Rao, N. N.; Rao, L. C.; Kumar, N. S. Tetrahedron Lett. 2012, 53, 3963.

[7] (a) Xie, J.; Li, H. M.; Zhou, J. C.; Cheng, Y. X.; Zhu, C. J. Angew. Chem., Int. Ed. 2012, 51, 1252.

(b) Iglesias, A.; Alvarez, R.; Lera, A. R. D.; MuÇiz, K. Angew. Chem., Int. Ed. 2012, 51, 2225.

(c) Baudoin, O. Chem. Soc. Rev. 2011, 40, 4902.

(d) Lyons, T. W.; Sanford, M. S. Chem. Rev. 2010, 110, 1147.

(e) Ramirez, T. A.; Zhao, B. G.; Shi, Y. Chem. Soc. Rev. 2012, 41,
931.

(f) Tsai, C. C.; Shih, W. C.; Fang, C. H.; Li, C. Y.; Ong, T. G.; Yap, G. P. A. J. Am. Chem. Soc. 2010, 132, 11887.

(g) Nakao, Y.; Yamada, Y.; Kashihara, N.; Hiyama, T. J. Am. Chem. Soc. 2010, 132, 13666.

(h) Campos, K. R. Chem. Soc. Rev. 2007, 36, 1069.

(i) Tobisu, M.; Chatani, N. Angew. Chem., Int. Ed. 2006, 45, 1683.

(j) Jazzar, R.; Hitce, J.; Renaudat, A.; Kreutzer, J. S.; Baudoin, O. Chem. Eur. J. 2010, 16, 2654.

(k) Qian, B.; Guo, S. M.; Shao, J. P.; Zhu, Q. M.; Yang, L.; Xia, C. G.; Huang, H. M. J. Am. Chem. Soc. 2010, 132, 3650.

[8] (a) Qian, B.; Guo, S. M.; Xia, C. G.; Huang, H. M. Adv. Synth. Catal. 2010, 352, 3195.

(b) Rueping, M.; Tolstoluzhsky, N. Org. Lett. 2011, 13, 1095.

(c) Komai, H.; Yoshino, T.; Matsunaga, S.; Kanai, M. Org. Lett. 2011, 13, 1706.

(d) Qian, B.; Xie, P.; Xie, Y. J.; Huang, H. M. Org. Lett. 2011, 13, 2580.

(e) Yan, Y. Z.; Xu, K.; Fang, Y.; Wang, Z. Y. J. Org. Chem. 2011, 76,6849 .

(f) Qian, B.; Shi, D. J.; Yang, L.; Huang, H. M. Adv. Synth. Catal. 2012, 354, 2146.

(g) Komai, H.; Yoshino, T.; Matsunaga, S.; Kanai, M. Synthesis 2012, 44, 2185.

(h) Liu, J. Y.; Niu, H. Y.; Wu, S.; Qu, G. R.; Guo, H. M. Chem. Commun. 2012, 48, 9723.

(i) Yang, Y. Z.; Xie, C. S.; Xie, Y. J.; Zhang, Y. H. Org. Lett. 2012, 14, 957.

(j) Guan, B. T.; Wang, B. L.; Nishiura, M.; Hou, Z. M. Angew. Chem., Int. Ed. 2013, 52, 4418.

[9] Wang, L.; Yao, Z. G.; Xu, F.; Shen, Q. Heteroat. Chem. 2012, 23, 449.

[10] Raghu, M.; Rajasekhar, M.; Reddy, B. C. O.; Reddy, C. S.; Reddy, B. V. S. Tetrahedron Lett. 2013, 54, 3503.

(Li, L.; Fan, Y.) 